\title{
\begin{tabular}{ll} 
Research Square & Preprints are preliminary reports that have not undergone peer review. \\
\hline & They should not be considered conclusive, used to inform clinical practice,
\end{tabular} or referenced by the media as validated information.
}

\section{Deep learning-based methods for forecasting Brent Crude Oil return considering COVID-19 pandemic effect}

\section{Pouya Khodaee}

Amirkabir University of Technology Department of Industrial Engineering and Management Systems

Seyed Mehrzad Asaadi Sajadi

Amirkabir University of Technology Department of Industrial Engineering and Management Systems

Ehsan Hajizadeh ( $\nabla$ ehsanhajizadeh@aut.ac.ir)

Amirkabir University of Technology https://orcid.org/0000-0003-4141-2151

\section{Sabri Farhadi}

Amirkabir University of Technology Department of Industrial Engineering and Management Systems

\section{Research Article}

Keywords: Deep Learning, CNN, LSTM, Covid-19, Energy Market

Posted Date: February 25th, 2022

DOI: https://doi.org/10.21203/rs.3.rs-1088913/v1

License: (c) (i) This work is licensed under a Creative Commons Attribution 4.0 International License.

Read Full License 


\title{
Deep learning-based methods for forecasting Brent Crude Oil return considering COVID-19 pandemic effect
}

\author{
Pouya Khodaee ${ }^{1}$, Seyed Mehrzad Asaadi Sajadi ${ }^{1}$, Ehsan \\ Hajizadeh $^{{ }^{*}}$ and Sabri Farhadi ${ }^{1}$
}

${ }^{1}$ Department of Industrial Engineering and Management Systems, Amirkabir University of Technology, Tehran, Iran.

\author{
*Corresponding Author E-mail: ehsanhajizadeh@aut.ac.ir; \\ Contributing Authors' E-mails: pouyakhodaee@aut.ac.ir (P. \\ Khodaee); mehrzad.sajadi@aut.ac.ir (S.M.A. Sajadi); \\ sabrifarhadi@aut.ac.ir (S. Farhadi);
}

\begin{abstract}
Forecasting return and profit is a primary concern for financial practitioners. It is even more critical when it comes to forecasting energy market returns. This research attempts to propose an effective method for predicting the Brent Crude Oil return, which results in remarkable performance compared to the well-known models in the return prediction. The proposed model is a hybrid model based on LSTM and CNN networks where ARIMA and GARCH outputs are used as features along with return lags, price, and macroeconomic variables for training the models, resulting in significant improvement in the model's performance. According to the obtained results, our proposed model performs better than other models, including ANN, PCA-ANN, LSTM, CNN. In the second part of this study, by considering the spread of the COVID-19 and its impact on the financial markets, we present a precise LSTM model that can reflect this disease's impact on the Brent Crude Oil return. This paper uses the significance test and correlation measures to show similarity between the series of Brent Crude Oil during the SARS period and COVID-19; after that, we use SARS period data along the COVID-19 to train the LSTM. The results demonstrate that the proposed LSTM model, tuned by the SARS data, can better predict the Brent Crude Oil return during the COVID19 pandemic.
\end{abstract}

Keywords: Deep Learning, CNN, LSTM, Covid-19, Energy Market 


\section{Introduction}

A very challenging issue in financial markets is modeling and forecasting the market's future. Because many economic goals are influenced by the future returns of financial markets, forecasting the market's return is of utmost importance for making the right investment decision. Predicting the financial market's future directions generally requires examining several forecasting modules, risk analysis, and trading strategy. One of the major financial markets to be addressed is the trend of oil return and price in the future, which significantly impacts the global economy. Some recent studies have demonstrated the importance of crude oil price or return prediction (Deng et al, 2020; Quayyoum et al, 2020; Wang and Wang, 2020; Zhang and Wang, 2021). This paper aims to forecast Brent Crude Oil's return accurately by using deep learning methods. Given that this natural substance has a tremendous impact on the decisions of many large financial institutions such as banks, manufacturing companies, and refining industries, it is, thus, necessary to predict the future trend of oil prices or their returns accurately.

For assessing financial volatilities, the parametric models have been developed with the advent of ARCH (Autoregressive Conditional Heteroscedasticity) and GARCH (Generalized ARCH) models presented by Engle (1982); Bollerslev (1986). Although financial time series have complex and nonlinear structures, linearity correlation structure is presumed in the classical models. Hence, they may not fit nonlinear structured time series, and considering linearity assumption can affect the model entirely. But using them as an input for feeding models, including ANN, can improve their performance (Kristjanpoller and Minutolo, 2015). Hajizadeh et al (2019) have improved the model's accuracy by using hybridization of GARCH models and neural networks for predicting the Euro/Dollar volatility exchange rate. Since volatility is one of the most influential parameters on the return, using GARCH models' output as a new input for forecasting the return could help the model train better.

Non-parametric models implemented by various approaches such as machine learning and deep learning can fit much better with the data set than classical linear models (Yu et al, 2009; Lu et al, 2009). For many years, experts have developed extended endeavors to take advantage of AI (Artificial Intelligence) to set up a system that assists traders with decision-making. Racine (2001) showed that neural network-based optimization models, a branch of artificial intelligence, can perform far better and maintain flexibility simultaneously. Hajizadeh et al (2012) applied a hybrid model based on EGARCH ${ }^{1}$ and ANN to predict the stock index volatility of the S\&P 500. Among other modern deep learning methods, CNN is mainly used for automatic feature selection and market forecasting. Like the ANN, CNN is based on artificial intelligence. Di Persio and Honchar (2016) presented a neural network approach for forecasting the stocks' trend. Furthermore, the CNN-based models' results have

\footnotetext{
${ }^{1}$ Exponential Generalized Autoregressive Conditional Heteroscedasticity
} 
been compared with different methods where CNN has shown remarkable performance. Because the long-term prediction of time series data is challenging, Long Short-Term Memory (LSTM) is another approach that can be used to maintain long-term dependencies. Wu et al (2018) used the LSTM method to forecast the price of Bitcoin. In a similar study, Karakoyun and Cibikdiken (2018) compared this method with the ARIMA method in predicting the price of Bitcoin.

This research attempts to provide a hybrid model with LSTM and CNN to take advantage of each to predict the future return of Brent Crude Oil. We also try to illustrate the presented models' superiority by comparing different up-to-date models described in the coming sections. Because of the data lack, after the outbreak of the COVID-19, SARS data are added to the COVID-19 data for better training of the neural networks. It is assumed, initially, and then is proved that the hypothesis of COVID-19 behavior would be similar to that of SARS. Therefore, SARS data is added to compensate for the lack of data. Finally, Brent Crude Oil's return is forecasted by applying the LSTM model for after the COVID-19 pandemic regarding the concerns raised by Bildirici et al (2020); Dey et al (2021) and other developed studies.

The remainder of this paper is organized as follows: In section 2, this research discusses the literature review of the methods used in this research. Section 3 describes the methodology of the models that lead us to present our proposed model in section 4 . Sections 4 presents the comparative and proposed models, respectively. The data and its characteristics are investigated in section 5. Section 6 contributes to seeing how better our proposed model performs compared to benchmark models through empirical results. Finally, in section 7 , the results and the future direction are discussed.

\section{Literature Review}

This section first reviews the GARCH-based models such as GARCH, FuzzyGARCH, and ANN-GARCH that are used to predict the volatility of financial data. Then, it puts forth to review the up-to-data models in literature which have been utilized to forecast financial markets' return, price, and volatility.

\subsection{Classical Models}

The first approach, which is based on the data from financial time series with stochastic variance and offers volatility intervals instead of forecasting it precisely, is the $\mathrm{ARCH}^{2}$. This approach, first introduced by Engle (1982), has been in the literature for a long run. Bollerslev (1986) developed a generalized model called GARCH ${ }^{3}$. He was the first who has examined ARCH models in financial terms both empirically and theoretically (Bollerslev et al, 1992). Bauwens et al (2006) have employed multivariate GARCH models because the

\footnotetext{
${ }^{2}$ Autoregressive Conditional Heteroscedasticity

${ }^{3}$ Generalized ARCH
} 
financial volatilities move along time with assets and markets; thus, univariHong (2011) have shown that classical methods, such as the GARCH model, are mean-revert and usually built with close price data. Therefore, this may result in the negligence of the important daily price changes, leading to data loss and inefficiency. Hence, the Range-based Autoregressive model was introduced and expanded to pay off these weaknesses. Developing the issue, Wang et al (2012) have presented the efficient semi-parametric GARCH model for financial volatility. Maciel et al (2016) have presented Fuzzy-GARCH models in predicting and modeling financial volatility. Sadik et al (2019) presented the NA-GARCH model for forecasting the stock price volatility, which is the combination of the GARCH model by examining the effects of quantified news sentiment on the movement of stock prices. Finally, Naimy et al (2021) compared the accuracy of GARCH models in assessing the cryptocurrencies volatility. Many financial time series data show a nonlinear dependency structure, but in GARCH models, a linear correlation structure is generally assumed between time-series data; hence, these models do not usually record the nonlinear patterns. As a result, the approximate linear models obtained from them in complex problems may not be satisfactory.

\subsection{Artificial-Intelligence-Based Models}

Artificial-Intelligence-based models have demonstrated superb performance in modeling and forecasting return, price, and volatility. Racine (2001) have shown that neural network-based optimization models, as a type of artificial intelligence, can perform far better than the classic GARCH models and illustrates better flexibility. Artificial Neural Network (ANN) can be much more applicable and flexible when GARCH and ARCH models' output feed the networks to predict the volatility and return of the financial market. Hamid and Iqbal (2004) have developed a neural network-based approach to forecast the future price volatility of the S\&P 500. Pérez-Rodríguez et al (2005) have examined the ANN and $\mathrm{STAR}^{4}$ models for predicting the Spanish Stock Index. Wang (2009) have proposed a nonlinear neural network method to predict a stock index's selected price. In the same line, Yu et al (2009) have presented metamodeling neural networks in forecasting financial time series. Bildirici and Ersin (2009) have worked on predicting robust GARCH family models integrated with ANN and, then, using it for predicting Istanbul Stock Exchange's return. Roh (2007) has implemented three aggregated financial time series methods and artificial neural networks in the KOSPI $200^{5}$. Hajizadeh et al (2012) have applied a hybrid model based on EGARCH-ANN to forecast stock index volatility S\&P 500. On the same page, Adhikari and Agrawal (2014) have presented an approach that processes the linear part of the financial data set by the Random Walk model and the lasting nonlinear part through a set of ANN and Elman ANN (EANN) models. Finally, Kristjanpoller and Minutolo

\footnotetext{
${ }^{4}$ Smooth Transition AutoRegression

${ }^{5}$ Korea Composite Stock Price Index 200
} 
(2015) have investigated the combination of GARCH and ANN approaches in predicting gold price volatility. Mohammed et al (2020) have proposed an innovative fuzzy-EGARCH-ANN model to forecast the stock market volatility. highly nonlinear financial data compared to the EGARCH model.

Using Support Vector Machine (SVM) to forecast the financial time series, Tay and Cao (2001) have discovered that SVM performed better than the Back-Propagation Neural Network (BPNN). Tang et al (2009) examined the volatility prediction Wavelet-Support Vector Machine (W-SVM), which is a hybridization of SVM and Discrete Wavelet Transform (DWT), in predicting volatilities. Chen et al (2010) have studied the SVM based on GARCH models to predict volatilities. Zhiqiang et al (2013) have developed this model by predicting the financial time series through SVM models and Locality Preserving Projection (LPP) and by considering the Particle Swarm Optimization (PSO) algorithm. Geng et al (2016) have improved the PSO algorithm by developing the interval volatility prediction using SVM. The use of these algorithms increased the SVM capabilities. While Zhao et al (2019) have introduced a new approach to stock price prediction based on SVM and Singular Spectrum Analysis (SSA). Then, Lu et al (2009) employed the Support Vector Regression (SVR) method. Sun and Yu (2020) designed a two-step volatility prediction method from the combination of the SVR and the GARCH model.

Although extracting useful features from the financial market is one of the problems and challenges, Convolutional Neural Networks (CNN) have largely solved this problem. This method, among other modern methods, is mostly used for automatic feature selection and market forecasting. Like the ANN method, CNN is based on AI. This method was used by Lawrence et al (1997) to identify faces. Yang et al (2015) have applied the Deep Convolutional Neural Networks (DCNN) method for identifying human activities over multi-channel time series. Di Persio and Honchar (2016) have introduced an artificial neural network approach for forecasting the stock market indices to predict trends. As Gunduz et al (2017) employed, the CNN could use technical indicators for each type of sample; however, it could not consider correlations between stock markets as another possible source of information. Finally, Hoseinzade and Haratizadeh (2019) have introduced a CNN-based framework for collecting data from various sources such as different markets to extract features for predicting these markets' future.

Because the long-term prediction of time series is a very challenging issue, Long Short-Term Memory (LSTM) is another approach that can be used to maintain long-term dependencies. Wu et al (2018) used the LSTM method to predict the price of Bitcoin. In a similar study, Karakoyun and Cibikdiken (2018) compared this method with the ARIMA method in predicting the price of Bitcoin. Siami-Namini et al (2018) examined the ARIMA and LSTM methods in time-series predictions. Kim and Won (2018) predicted Korea Composite Stock Price Index 200 (KOSPI 200) stock market price volatility using LSTM models and GARCH. Cao et al (2019) used the Complete Ensemble Empirical 
Mode Decomposition with Adaptive Noise and the LSTM method to predict the financial time-series models. Tomar and Gupta (2020) also predicted the spread of the COVID-19 virus in China using the LSTM method. Finally, Livieris et al (2020) employed the CNN-LSTM hybrid method to forecast the gold market.

Classical methods have linear nature, and hence they are not suitable for employing in complex and non-linear situations. For addressing this problem, this study takes advantage of AI models. Furthermore, the GARCH and ARCH models' output feed the neural networks. Since the CNN model is very efficient in extracting valuable features from the primary data and the LSTM model helps identify short-term and long-term dependencies (Livieris et al, 2020), in this study, a combination of these two models is used to predict Brent Crude Oil prices.

\section{Methodology}

In this section, the CNN and the LSTM basic concepts will be discussed in detail. After explaining the CNN and LSTM separately, this study will present its hybrid model in the forthcoming section.

\subsection{CNN}

CNN is one of the Deep Learning models. This model's components are an input layer, convolutional layers, nonlinear activation function, pooling layers, and a fully connected layer. In most cases, the input layer data of this network is one-dimensional, two-dimensional, or three-dimensional. This paper converts the features of the time series of the Brent Oil into two-dimensional inputs to take advantage of the CNN network. The taken approach for converting them into $2 \mathrm{D}$ inputs will be presented in the coming sections.

\subsubsection{Convolutional Layers}

Convolution layers work by applying filters to each sample input and passing them to the next layer that is convoluted by filter size. These newly generated kernels containing valuable information obtained from the primary data will be passed input into the following layers. Finally, they produce a matrix with high-level features as an output of convolutional layers. The freshly obtained matrix is usually given as an input to the fully connected layer after having done the convolution process and passing through the Max Pooling layers. According to Krizhevsky et al (2012), the input data should have a matrix form; therefore, data processing is continuing by taking the two-dimensional image(matrices). Since convolution operations aim to extract high-level features, more than one convolution layer is typically used. Usually, the first layer of convolution extracts the low-level features, and by adding the subsequent layers of the network, it will be able to extract the high-level features. Hence, in the proposed model, the network uses two layers of convolution to extract 
high-level features. More precisely, as in Livieris et al (2020) was discussed, convolution layers convolute the input data. Thus, a convolution kernel, which in most cases has dimensions of $3 * 3$ or $5 * 5$, is used to apply to the initial matrix. The kernel of the convolution used in this model is $3 * 3$, which slides from the upper left of the image on the matrix, with a specified stride length (the stride length is one in this research). Each time the multiplication occurs across the kernels whose entries are updated and changed after each network training, this process continues until the kernel covers the whole input matrices. The padding technique is used in the CNN model of this research, which is a common technique to control the shrinkage of dimension after applying filters larger than $1^{*} 1$. Moreover, it avoids losing information at the boundaries (Li et al, 2020).

\subsubsection{Max Pooling Layer}

Like convolution layers, this layer is used to extract useful features from the convolved matrix, which is done by reducing the matrix's dimensions. This layer extracts the maximum amount of matrix elements that are covered each time by the kernel. This layer helps the network become more robust, the output of which will eventually be a matrix with lower dimensions.

\subsection{LSTM Model}

It is a branch of the recurrent neural network (RNN) model with the capacity of learning long-term dependencies. Sometimes we need past information and data trends to make a more reliable prediction. RNNs can use long-term information in processing current data. However, RNN can only learn a limited number of short-term dependencies, and at longer distances, the RNN networks lose their ability to recall past information. LSTM can be considered an extension to RNN that can connect the past information with the present ones and maintain the long-term data dependencies during data processing. While there is one processing layer in RNN, we have four processing units in LSTM. Each LSTM block consists of a memory cell, input, forget, and output gates. Gates are a way to enter information voluntarily. Gates in the LSTM network assist information processing with the Sigmoid activation function where the output is between 0 and 1. The Forget Gate controls the flow of information from the previous step, which determines whether to use the previous information and, if yes, how much of it should be affected in the next layers. The Input Gate controls the new information, which examines whether we use this current information in the process or not, and if so, how much of it should be used. Finally, the output Gate examines how much information from the previous and current time steps should be combined and transferred to the next step.

These features help LSTM with controlling the flow of information and with learning long-term dependencies (Sagheer and Kotb, 2019; Baek and Kim, 2018). Fig. 1 shows the architecture of an LSTM network block. The symbols 


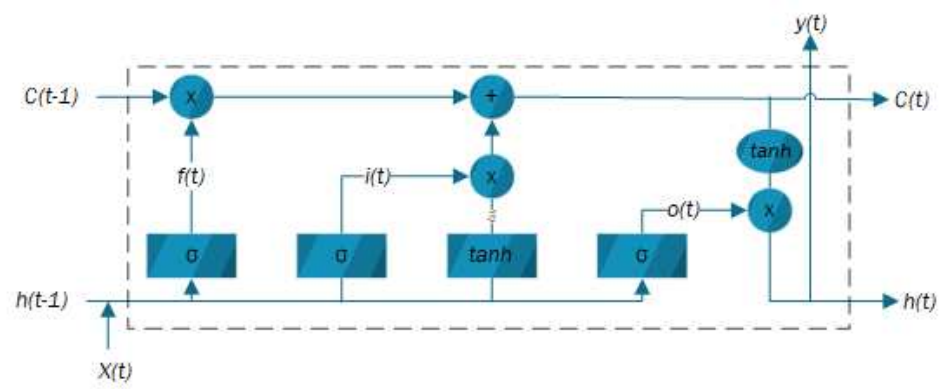

Fig. 1 Each unit of LSTM Architecture

used in the model and their definitions are presented in Table. 1. It is to be noted that $C_{t}$ and $h_{t}$ in each layer of LSTM are entered as input to the next layer. At Forget Gate, the $f_{t}$ function outputs is a number of zero or one, meaning which flow of information should be stopped or continued. A value of one means that it entirely moves the value of the $C_{t}-1$ state cell to $C_{t}$, and a value of zero means it clears the $C_{t}-1$ state cell information and does not take any of it to the $C_{t}$ state cell. Eq. 1 shows the calculation of each unit of LSTM:

$$
\begin{gathered}
i_{t}=\sigma\left(U_{i} x_{t}+W_{i} h_{t-1}+b_{i}\right) \\
f_{t}=\sigma\left(U_{g} x_{t}+W_{g} h_{t-1}+b_{g}\right) \\
c_{t} *=\tanh \left(U_{c} x_{t}+W_{c} h_{t-1}+b_{c}\right) \\
c_{t}=g_{t} \bigodot c_{t-1}+i_{t} \bigodot c_{t} * \\
o_{t}=\sigma\left(U_{o} x_{t}+W_{o} h_{t-1}+b_{o}\right) \\
h_{t}=o_{t} \bigodot \tanh \left(c_{t}\right)
\end{gathered}
$$

Table 1 LSTM Terms

\begin{tabular}{ll}
\hline Definition & Symbol \\
\hline The input gate & $i_{t}$ \\
The forget gate that controls the previous information & $f_{t}$ \\
The second gate that controls the new information & $C_{t}$ \\
The state of memory at time t & $C_{t}$ \\
Output gate which manages the information and could be used as the memory & $o_{t}$ \\
cell output & \\
The input & $x_{t}$ \\
The hidden state that constitutes the memory cell output & $h_{t}$ \\
Weight matrices & $U * \& W *$ \\
The bias term vectors & $B *$ \\
The Sigmoid function & $\sigma$ \\
The operator which component-wise multiplication & $\odot$ \\
\hline
\end{tabular}




\section{Comparative \& Proposed Models}

This section will first review the benchmark models used to compare the proposed model's performance. It then drives into detail the proposed model. In each sub-section, firstly, a brief review of the selected model will be given, then, the approach of this study to take advantage of them for forecasting the return is discussed.

\subsection{ANN \& ANN-PCA}

The ANN has contained a set of interconnected neurons in different layers that exchange information with each other. This network contains three primary layers: an input layer, hidden layers, and an output layer. In the input layer, each neuron's information, which is a vector, is connected to the hidden layer neurons by a connection (synapse). The random weights are assigned to each of the input layer neurons connecting to the hidden layer units. The input vectors are multiplied by each connection's corresponding weights, and the nonlinear activation function is used. Therefore, through backpropagation derivatives, the weights of the hidden layer components are updated. Then the output of all processing units from each layer is given as input to the next layer. If the number of layers is large, this process will be called Deep Neural Network (DNN).

In this study, the ANN model is used in a similar way to the proposed models in Hajizadeh et al (2012). The PCA is applied to reduce the size of the input dataset so that it does not omit any variables but will generate new variables with more important information achieving from original inputs. Another advantage is that the new variables are independent and have no correlation (Abdi and Williams, 2010). In this study, we choose two Principal Components that can describe the 0.95 of data variability. The PCA used in this study is similar to that used by Chen and Hao (2018). Consequently, the ANN is used to predict oil return, then in the second method, we used the PCA technique and its output with the ANN network. Zhong and Enke (2017)'s model is used to implement the hybrid PCA-ANN method.

\subsection{CNN-ANN \& LSTM}

Another comparative model is the CNN-ANN hybrid model, which is based on the model developed by Haggag et al (2019). The CNN network's output is inputted to the ANN part of model containing fully connected layers. The first fully connected layer has 200 neurons, and the second dense layer contains 100 neurons up to the fourth layer with 60, 10 neurons. Each layer uses the RELU activation function, after that output of the CNN-ANN hybrid model is obtained. Fig. 2 shows the CNN-ANN architecture which is used. Drawn upon the studies by Sagheer and Kotb (2019) for before COVID-19 data and Tomar and Gupta (2020) for COVID-19 data, the LSTM method was implemented. 


\section{CNM}

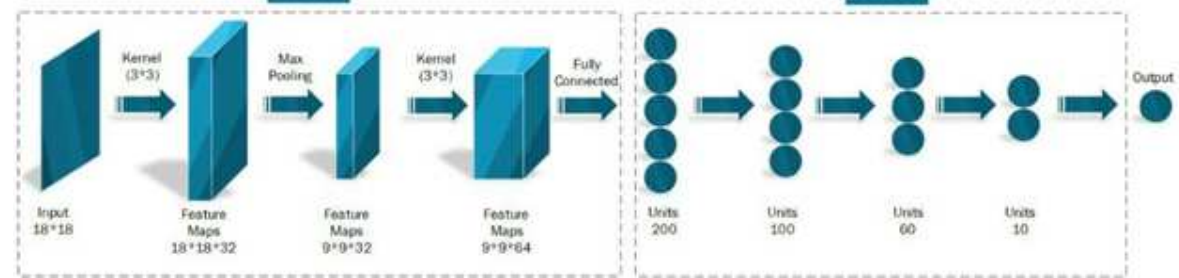

Fig. 2 Proposed CNN-ANN Model Architecture

\subsection{Proposed 2D CNN-LSTM}

In the following, the proposed 2D CNN-LSTM is explained.

At first, the inputs are converted into $2 \mathrm{D}$ images as well as in the CNNANN model. The approach for converting them is made by considering every 18 days as the rows of the matrix and the features as the columns. As mentioned above, each $18 * 18$ is considered an image (matrix). As a result, these inputs feed the models. The CNN model is very efficient in extracting useful features from the primary data; on the other hand, the LSTM model is useful for detecting long-term and short-term dependencies (Livieris et al, 2020). This study combines these two models with considering both models' capabilities in predicting Brent Crude Oil return. Our proposed model is based on the CNN-LSTM hybrid model. We use two layers of convolution and one layer of Max Pooling. The first layer of convolution consists of 32 filters, and the second layer of convolution is used with 64 filters. The CNN network outputs, which are valuable features, are connected to the fully connected layer, and this layer output is given as a new input to the LSTM network. The output of this network is then given to another fully connected layer, and finally, the 2D-CNN-LSTM output will be one dimension. Fig. 3 illustrates the flowchart of our study's main steps. Fig. 4 illustrates the hybrid model of these two networks. Table. 2 shows the information about the characteristics of each of these methods. Python implements the models, and the library used in the coding deep networks is PyTorch.

\section{Characteristics of the Data}

In this study, we have collected Brent Crude Oil's daily prices from February 17, 2013, to December 30, 2019, before COVID-19, and from December 31, 2019, to September 24, 2020, after the outbreak of the pandemic. We also accumulate Severe Acute Respiratory Syndrome (SARS) data from November 18, 2002, to May 19, 2004. All data was collected from Investing and Yahoo Finance databases. This study uses time-series data to predict Brent Crude Oil return. The input data are normalized regarding Eq. 2. Table. 3 shows the statistical characteristics of the series.

$$
x_{n o r m}=\frac{x-x_{\min }}{x_{\max }-x_{\min }}
$$




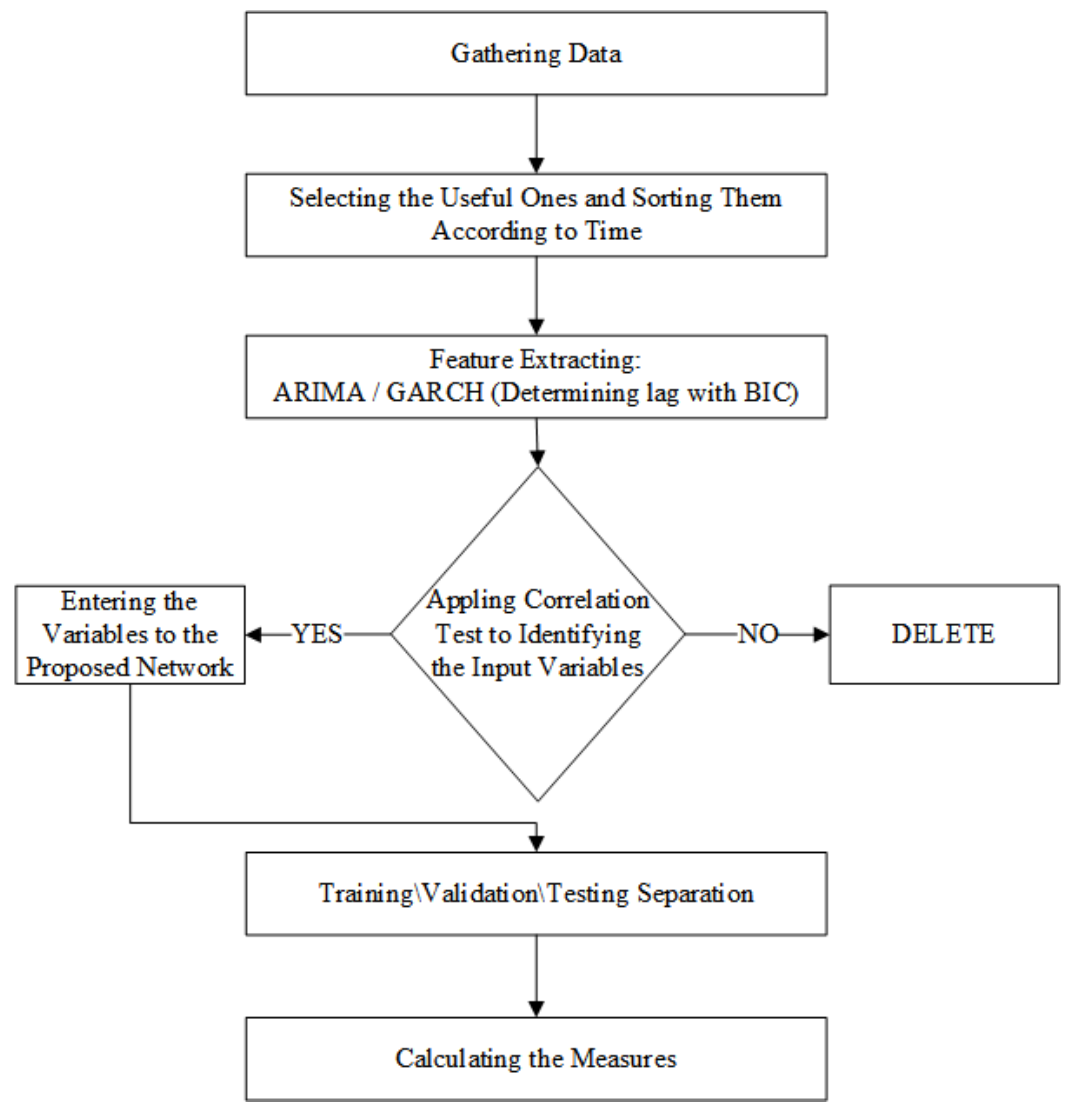

Fig. 3 Flowchart of main steps

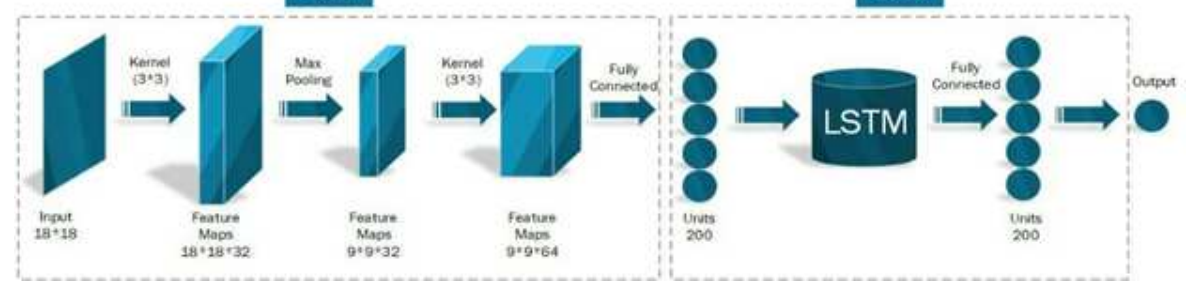

Fig. 4 Proposed 2D-CNN-LSTM Model Architecture

Table. 4 demonstrates the features used in this study before the outbreak of COVID-19. As different markets affect each other, and there is a high correlation between them, we used features such as exchange rates, crude oil, and natural gas prices to forecast Brent Crude Oil return. It should be noted that GARCH and ARIMA's outputs are used as inputs of our models. For the selected period, the return changes have been illustrated in Fig. 5. 
Deep learning-based methods for forecasting Brent Crude Oil return ...

Table 2 Models Information

Five computational measures, including Mean Square Error (MSE), Root Mean Square Error (RMSE), Mean Absolute Percentage Error (MAPE), Mean Forecast Error (MFE), and Mean Absolute Error (MAE), are applied to evaluate the efficiency of the models in forecasting the future return of Brent Crude Oil. The formula of each measures is shown in Table. 5. These measures demonstrate the error each time that the neural networks are executed. When the neural networks are run for the first time, the model assigns initial values with the random weight and the bias vectors. Then, the neural network calculates its error at each execution and updates the error values, weights, and biases by minimizing the derivatives.

\section{Empirical Results}

This section reports the results and outputs of the ANN, PCA-ANN, CNNANN, LSTM, and 2D-CNN-LSTM models. The information of implementing these methods is provided in Table. 2, which have been explained in the previous sections. Because each trader can choose different horizons for their trades, 
Table 3 Data characteristics

we decided to choose our models' forecast horizon for the next one day and the next five days to address this issue.

\subsection{Before the COVID-19 Pandemic}

The pre-COVID-19 data are divided into three segments: train, crossvalidation, and test data. It included 1795 data observations that 1500 are used for train and cross-validation data and the observations of 1501-1795 for test data. Table. 6 displays the results of the one-day-ahead forecast horizon. Table. 6 shows that the 2D-CNN-LSTM method has a far better forecasting performance than other models in terms of evaluation measures. The 2D-CNNLSTM has the lowest values on MSE, RMSE, MAE, MAPE, and MFE with values of $0.00014,0.01217,0.00387,0.13008,0.0017$, respectively. According to Table. 6, after the 2D-CNN-LSTM model, the LSTM and the CNN-ANN models provide better performance than other models. As was mentioned earlier, we used PCA to reduce the dimensions. The principle component is derived from the linear combination of input data and the new space direction matrix. We set the Principle Components (PCs) number to 18 in this study for the PCA-ANN model. Table. 7 presents the explained PCs' variance in descending order, the first PC describing the most variability. Fig. 6 illustrates that PC 1 and PC 2 could describe approximately $95 \%$ of the data volatility. 
Deep learning-based methods for forecasting Brent Crude Oil return ...

Table 4 Selected Features as the inputs of the models

\begin{tabular}{llll}
\hline 1 & Price Normalized & 10 & 8-days lag-return \\
\hline 2 & Return & 11 & 9-days lag-return \\
\hline 3 & 1-day lag-return & 12 & 10-days lag-return \\
\hline 4 & 2-days lag-return & 13 & Index EURO/Dollar \\
\hline 5 & 3-days lag-return & 14 & Price Natural Gas \\
\hline 6 & 4-days lag-return & 15 & US dollar index \\
\hline 7 & 5-days lag-return & 16 & Price Crude Oil \\
\hline 8 & 6-days lag-return & 17 & ARIMA(4,0,3) \\
\hline 9 & 7-days lag-return & 18 & GARCH(4,3) \\
\hline
\end{tabular}

Table 5 The Computational Measures' Formula

\begin{tabular}{lll}
\hline Evaluation Criteria & Formula & Descriptions \\
\hline MSE & $\frac{\sum_{v=1}^{n}\left(A_{v}-F_{v}\right) 2}{n}$ & \\
RMSE & $\left(\frac{\sum_{v=1}^{n}\left(A_{v}-F_{v}\right) 2}{n}\right)^{\frac{1}{2}}$ & $n=$ number of total iteration each run \\
MAE & $\frac{\sum_{v=1}^{n}\left|A_{v}-F_{v}\right|}{n}$ & $A_{v}=$ actual value \\
MAPE & $\frac{\sum_{v=1}^{n}\left|\frac{A_{v}-F_{v}}{A_{v}}\right|}{n}$ & $F_{v}=$ forecast value \\
MFE & $\frac{\sum_{i=1}^{n}\left(e_{i}\right)}{n}$ & \\
\hline
\end{tabular}

It was also noticed that in using PC 1 and PC 2 , about $0.0084 \%$ of data is lost. This error rate was calculated through Eq. 3. The use of PCA besides ANN in forecasting the oil return on a one-day ahead forecasting has greatly improved the forecasting accuracy and brought the forecast return closer to the actual value. The observed values for each of the five evaluation measures used in the PCA-ANN model are lower than those in the ANN model. As Table. 8 demonstrates, we considered the forecast horizon for five days. According to the obtained results, the proposed model, namely 2D-CNN-LSTM, also had higher efficiency compared to other models. As the observations indicate, 2DCNN-LSTM had the lowest values in MSE RMSE, MAE, MAPE, with the values of $0.00014,0.01187,0.00326,0.10232$, respectively. Like one-day ahead forecast horizon, in the five-day ahead forecast horizon, after the 2D-CNNLSTM model, the LSTM model and then CNN-ANN had better performances. Unlike the one-day ahead forecasting horizon where PCA's use improved the 
ANN model's performance, the use of PCA in the five-day ahead forecasting did not work well. The prediction using the ANN model had a better performance without the use of PCA.

$$
\frac{\frac{1}{m} \sum_{i=1}^{m}\left\|x^{(i)}-x_{\text {approx }}^{(i)}\right\|^{2}}{\frac{1}{m} \sum_{i=1}^{m}\left\|x^{(i)}\right\|^{2}} \leqslant 0.01
$$

Regarding the observed values of the models implemented in this study to predict the return of Brent Crude Oil before the outbreak of the COVID-

Table 7 The Degree of Variability Explained by PCs

\begin{tabular}{lccccc}
\hline & ANN & PCA-ANN & CNN-ANN & LSTM & 2D CNN-LSTM \\
\hline MSE & 0.00039 & 0.00031 & 0.0003 & 0.0002 & 0.00014 \\
RMSE & 0.01905 & 0.01749 & 0.01735 & 0.0149 & 0.01217 \\
MAE & 0.0146 & 0.01355 & 0.0132 & 0.005 & 0.00387 \\
MAPE & 2.3443 & 1.49995 & 2.053 & 0.207 & 0.13008 \\
MFE & -0.00697 & -0.0078 & 0.0081 & 0.0086 & 0.0017 \\
\hline
\end{tabular}
19 pandemic, it can be stated that the models outperformed in the five-day ahead forecast horizon. The values for MSE are 0.00014, 0.00014 for the oneday ahead and five-day ahead forecast respectively, 0.01217, 0.01187 for the RMSE, 0.00387, 0.00326 for the MAE, 0.13008, 0.10232 for the MAPE, and finally $0.0017,0.0010$ for the MFE.

Table 6 The Amount of Each Measure for pre-COVID-19 with One-Day Ahead Forecast

\begin{tabular}{cccc}
\hline & & & \\
& Explained Variance & PCs & Explained Variance \\
\hline 1 & 0.9453 & 10 & 0.00031 \\
2 & 0.01849 & 11 & 0.00018 \\
3 & 0.01037 & 12 & 0.00009 \\
4 & 0.00877 & 13 & 0.00006 \\
5 & 0.00737 & 14 & 0.000056 \\
6 & 0.00471 & 15 & 0.000046 \\
7 & 0.00152 & 16 & 0.000041 \\
8 & 0.00132 & 17 & 0.000039 \\
9 & 0.00126 & 18 & 0.0000069 \\
\hline
\end{tabular}

Fig. 7 shows the Brent Crude Oil return forecast using the ANN method with the one and five-day ahead forecast horizons. Fig. 8 illustrates Brent Crude Oil return forecast using PCA-ANN. Fig. 9 also demonstrates the output of the CNN-ANN method. Finally, Fig. 10 displays LSTM, and Fig. 11 illustrates 2D-CNN-LSTM that predict the future return of Brent Crude Oil in one and five-day ahead forecasting horizons. 
Table 8 The Amount of Each Measure for pre-COVID-19 with Five-Day Ahead Forecast

\begin{tabular}{lccccc}
\hline & ANN & PCA-ANN & CNN-ANN & LSTM & 2D CNN-LSTM \\
\hline MSE & 0.00031 & 0.00031 & 0.00027 & 0.0002 & 0.00014 \\
RMSE & 0.01766 & 0.01773 & 0.01652 & 0.01415 & 0.01187 \\
MAE & 0.01346 & 0.01448 & 0.00663 & 0.00484 & 0.00326 \\
MAPE & 1.55111 & 1.926 & 1.05369 & 0.1495 & 0.10232 \\
MFE & 0.00019 & 0.00222 & 0.00012 & 0.0017 & 0.001 \\
\hline
\end{tabular}

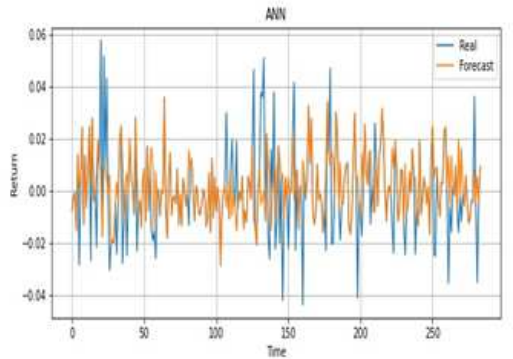

(a)

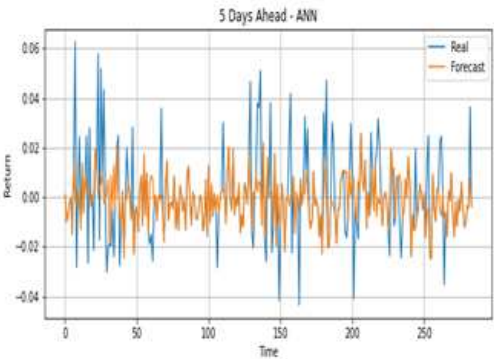

(b)

Fig. 7 ANN Forecasting Result for One-Day (a) and Five-Day (b) Ahead

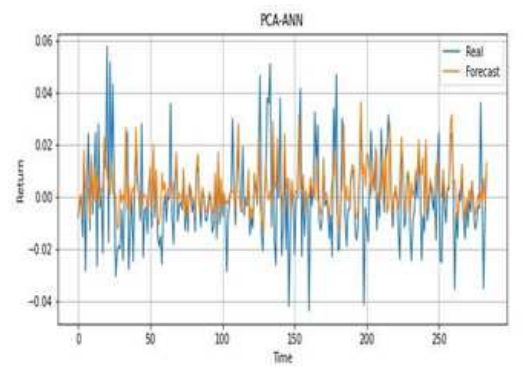

(a)

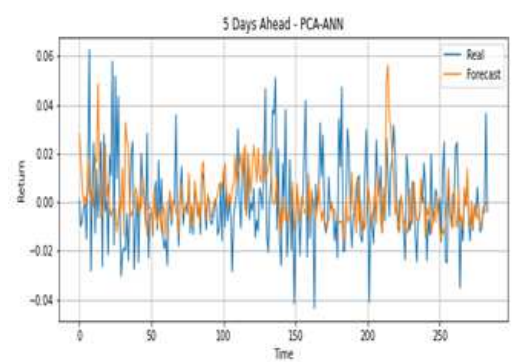

(b)

Fig. 8 PCA-ANN Forecasting Result for One-Day (a) and Five-Day (b) Ahead 


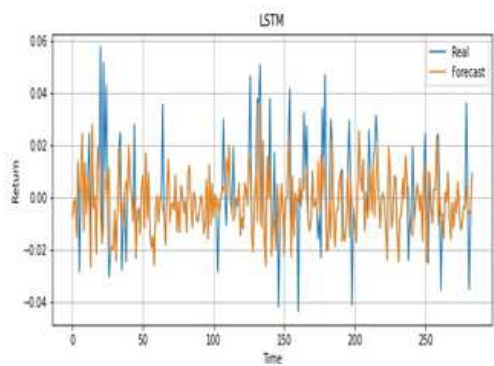

(a)

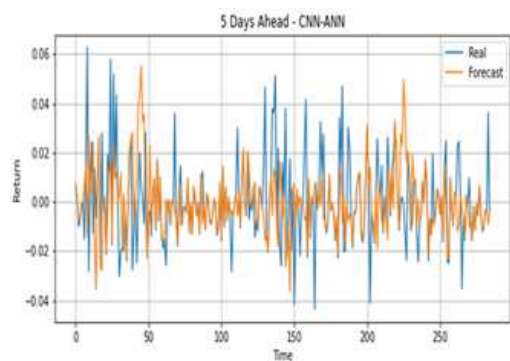

(b)

Fig. 9 CNN-ANN Forecasting Result for One-Day (a) and Five-Day (b) Ahead

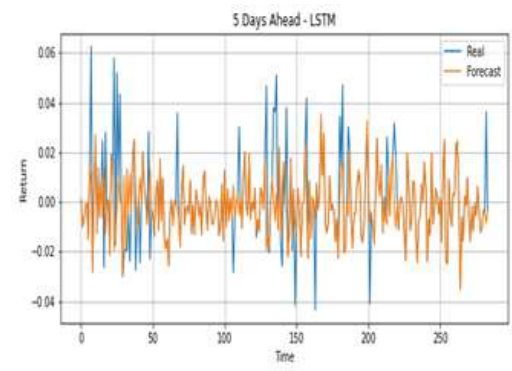

(b)

Fig. 10 LSTM Forecasting Result for One-Day (a) and Five-Day (b) Ahead

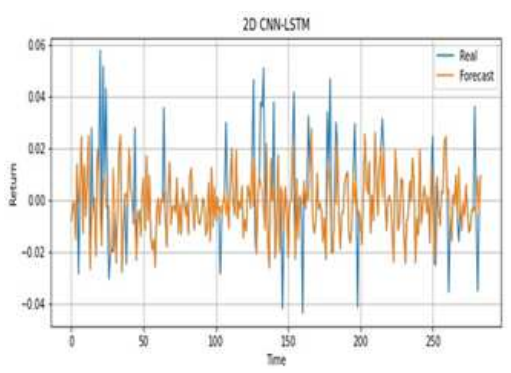

(a)

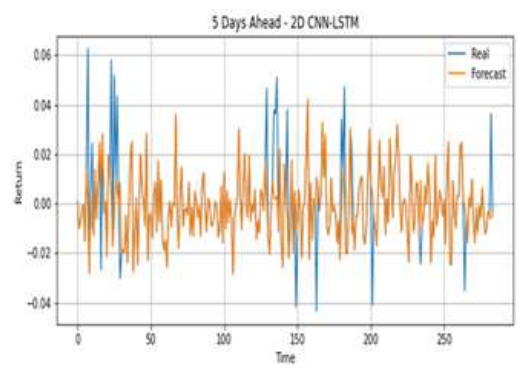

(b)

Fig. 11 2D CNN-LSTM Forecasting Result for One-Day (a) and Five-Day (b) Ahead

\subsection{During COVID-19 Outbreak}

Firstly, during the COVID-19 outbreak, which has 188 observations, data are divided into train and test data. Using the sliding window technique, the data are converted into a series of dependent and continuous sequences. Because this study considered the window size of 20 , the number of sets of independent series became 168 . 


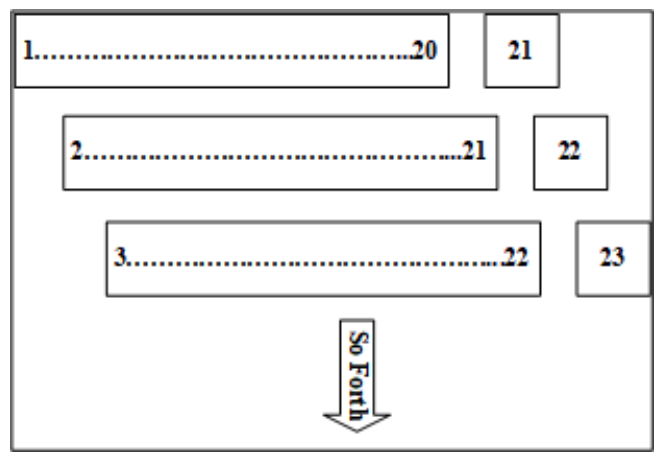

Fig. 12 Process of Sliding Window

Because of the lack of data during the outbreak of the COVID-19 pandemic, this study used SARS data. It is demonstrated that COVID-19 behavior would be similar to the SARS pandemic. Therefore, this study tries to select a portion of the SARS data that has so far behaved more like COVID-19. First, this research separates the oil returns during the SARS period into sets in which the length of each set is 188 because the number of observations for the COVID19 is 188. Therefore, 300 SARS data are separated into 188 observation-length data series, and then this research examines the correlation between each of the SARS-derived sets and the 188 COVID-19 series. The Pearson Correlation Coefficient test is used to investigate the relationship between SARS and the COVID-19 data series. This research considers the null hypothesis of the test to be no correlation between the series. The alternative hypothesis is nonzero of this correlation at a confidence level of $95 \%$. After calculating the correlation between all SARS data series and the COVID-19 data series, the SARS data series could not reject the null hypothesis at the significance level remain, and the rest are left. Then, this research keeps the series with the highest correlation with the COVID-19 data series from the remaining data series. The results obtained from this test show that the highest correlation coefficient is the 60 th data series with a correlation equal to 0.7 . According to the description, set 60 is selected. Then, this study separated the data from 60 to 300 from SARS and used it as an aid for network training. Moreover, the sliding window is applied to convert the data into different segments.

The window size used in this study is 20, in that of 188 COVID-19 data records used in network training. $X_{i}$ to $X_{i+19}$ data with $i=1,2, \ldots, 168$ are utilized to predict the next day return as the response variable $Y_{i+20}$. For instance, when we consider $i$ equal 1 , data $X_{1}$ to $X_{20}$ are selected to predict $Y_{21}$ (the return of day 21). Fig. 12 shows the sliding window process with a window size of 20 .

The effect of COVID-19 on oil return is similar to SARS regarding the above descriptions. Because the spread of COVID-19 is faster than that of SARS, this paper decided to use the SARS data besides the COVID-19 data 
in the network training. Therefore, the 220 SARS data sets and 130 COVIDfor network testing.

According to the previously introduced measures, we appraised the performance of the model. Considering the above explanations, we compared the COVID-19 and SARS data with the forecast horizons of one and five days in network training. Given the observations shown in Table. 9, which is related to the one-day ahead forecast horizon, the results demonstrate that the LSTM model (SARS+COVID-19) performs better than the LSTM (COVID-19) model. The LSTM model (SARS+COVID-19) and the LSTM model (COVID-19) have the values 0.00023 and 0.00050 for the MSE, 0.01527 and 0.02237 for RMSE, 0.011, 0.01585 for MAE, 2.2044, 2.7714 for MAPE, and $0.0067,-0.0056$ for MFE. Given these values, it can be deduced that the performance of the LSTM network (SARS + COVID-19) for predicting Brent Crude Oil return has increased significantly and has improved the network performance.

Table 9 The Value of Each Measure for COVID-19 Period with One-Day Ahead Forecast

\begin{tabular}{lcc}
\hline & LSTM $($ SARS+COVID-19) & LSTM (COVID-19) \\
\hline MSE & 0.00023 & 0.0005 \\
RMSE & 0.01527 & 0.02237 \\
MAE & 0.011 & 0.01585 \\
MAPE & 2.2044 & 2.7714 \\
MFE & 0.0067 & -0.0056 \\
\hline
\end{tabular}

Table. 10 shows the five-day-ahead forecast horizon. LSTM model $(\mathrm{SARS}+\mathrm{COVID}-19)$ and LSTM model (COVID-19) values are 0.00031, 0.00033 for MSE, 0.01773, 0.01840 for RMSE, 0.01448, 0.0143 for MAE, 1.926, 2.830 for MAPE, and 0.00222 and $-8.55 \mathrm{e}-05$ for MFE. Given these values, the LSTM network (SARS COVID-19) in predicting Brent Crude Oil return in three criteria of MSE, RMSE, and MAPE has a better performance than the other model. Based on the observed values, the performance of LSTM $(\mathrm{SARS}+\mathrm{COVID}-19)$ in the one-day ahead forecast horizon is better than the five-day ahead forecast horizon.

Table 10 The Value of Each Measure for COVID-19 Period with Five-Day Ahead Forecast

\begin{tabular}{lcc}
\hline & LSTM (SARS+COVID-19) & LSTM (COVID-19) \\
\hline MSE & 0.00031 & 0.00033 \\
RMSE & 0.01773 & 0.0184 \\
MAE & 0.01448 & 0.0143 \\
MAPE & 1.926 & 2.83 \\
MFE & 0.00222 & $-8.55 \mathrm{E}-05$ \\
\hline
\end{tabular}




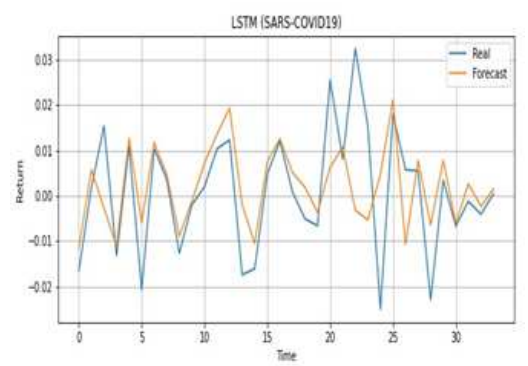

(a)

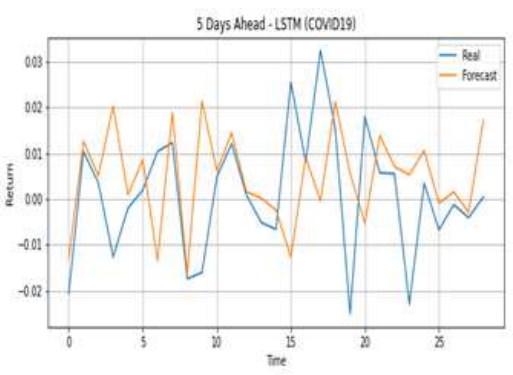

(b)

Fig. 13 LSTM-COVID-19 Forecasting Result After COVID-19 Pandemic with One-Day (a) and Five-Day (b) Ahead Forecast

Fig. 14 LSTM (SARS+COVID-19) Forecasting Result After COVID-19 Pandemic with One-Day (a) and Five-Day (b) Ahead Forecast

Fig. 13 shows Brent Crude Oil return's prediction using the LSTM method with one and five-day ahead Forecast horizons during the outbreak of the COVID-19 pandemic. Fig. 14 demonstrates the diagram of the return for LSTM (SARS +COVID-19). The difference of this method with simple LSTM is the consideration of oil price return data during the SARS outbreak, which is considered as an input in the implementation of the network and has significantly improved the network performance of the model.

\section{Conclusion}

In this study, the problem of forecasting future returns of Brent Crude Oil has been investigated. To do this, a new hybrid CNN-LSTM to take advantage of each of them has been proposed. Moreover, to enhance the performance of proposed models, the outputs of calibrated ARIMA and GARCH models, along with other variables such as return lags, price, and macroeconomic variables, have been considered as explanatory features for training the models. According to the obtained results and performance measures, our proposed model performs better than other models existing in the literature, including ANN, 
PCA-ANN, LSTM, CNN-ANN. Furthermore, for tuning the proposed models and increasing their efficiency during the COVID-19 pandemic, we use the SARS pandemic data that has a high correlation with COVID-19 data. The results demonstrate that the proposed LSTM model, tuned by the SARS data, can better predict the Brent Crude Oil return during the COVID19 pandemic. Using the accurate forecasts of crude oil return for various economic decisionmaking problems such as real options valuation, portfolio optimization, and 


\section{Author Contributions}

- Pouya Khodaee: Methodology, Software implementation, Visualization Analyzing, Investigation.

- Seyed Mehrzad Asaadi Sajadi: Software implementation, Visualization

- Ehsan Hajizadeh: Supervision, Conceptualization, Methodology, Data curation, Writing- Reviewing and Editing

- Sabri Farhadi: Visualization, Writing- Reviewing

\section{Ethical Approcal}

This article does not contain any studies with human participants or animals performed by any of the authors. All the data have been gathered from the open source sits, their names were mentioned in the passage.

\section{Funding Details}

The authors declare that this paper was not funded by either an institution or by a person.

\section{Declarations}

The authors have no conflicts of interest to declare that they are relevant to the content of this article.

\section{References}

Abdi H, Williams LJ (2010) Principal component analysis. Wiley interdisciplinary reviews: computational statistics 2(4):433-459

Adhikari R, Agrawal R (2014) A combination of artificial neural network and random walk models for financial time series forecasting. Neural Computing and Applications 24(6):1441-1449

Baek Y, Kim HY (2018) Modaugnet: A new forecasting framework for stock market index value with an overfitting prevention lstm module and a prediction lstm module. Expert Systems with Applications 113:457-480

Bauwens L, Laurent S, Rombouts JV (2006) Multivariate garch models: a survey. Journal of applied econometrics 21(1):79-109

Bildirici M, Ersin ÖÖ (2009) Improving forecasts of garch family models with the artificial neural networks: An application to the daily returns in istanbul stock exchange. Expert Systems with Applications 36(4):7355-7362

Bildirici M, Guler Bayazit N, Ucan Y (2020) Analyzing crude oil prices under the impact of covid-19 by using lstargarchlstm. Energies 13(11):2980 
Bollerslev T (1986) Generalized autoregressive conditional heteroskedasticity. Journal of econometrics 31(3):307-327

Bollerslev T, Chou RY, Kroner KF (1992) Arch modeling in finance: A review of the theory and empirical evidence. Journal of econometrics 52(1-2):5-59

Cao J, Li Z, Li J (2019) Financial time series forecasting model based on ceemdan and lstm. Physica A: Statistical Mechanics and its Applications 519:127-139

Chen S, Härdle WK, Jeong K (2010) Forecasting volatility with support vector machine-based garch model. Journal of Forecasting 29(4):406-433

Chen Y, Hao Y (2018) Integrating principle component analysis and weighted support vector machine for stock trading signals prediction. Neurocomputing 321:381-402

Deng S, Xiang Y, Nan B, et al (2020) A hybrid model of dynamic time wrapping and hidden markov model for forecasting and trading in crude oil market. Soft Computing 24(9):6655-6672

Dey P, Saurabh K, Kumar C, et al (2021) t-sne and variational auto-encoder with a bi-lstm neural network-based model for prediction of gas concentration in a sealed-off area of underground coal mines. Soft Computing pp 1-25

Di Persio L, Honchar O (2016) Artificial neural networks architectures for stock price prediction: Comparisons and applications. International journal of circuits, systems and signal processing 10(2016):403-413

Engle RF (1982) Autoregressive conditional heteroscedasticity with estimates of the variance of united kingdom inflation. Econometrica: Journal of the econometric society pp 987-1007

Geng L, Liang Y, Zhang Z, et al (2016) Forecasting range volatility using support vector machines with improved pso algorithms. Telkomnika 14

Gunduz H, Yaslan Y, Cataltepe Z (2017) Intraday prediction of borsa istanbul using convolutional neural networks and feature correlations. KnowledgeBased Systems 137:138-148

Haggag M, Abdelhay S, Mecheter A, et al (2019) An intelligent hybrid experimental-based deep learning algorithm for tomato-sorting controllers. IEEE Access 7:106,890-106,898

Hajizadeh E, Seifi A, Zarandi MF, et al (2012) A hybrid modeling approach for forecasting the volatility of s\&p 500 index return. Expert Systems with 
Applications 39(1):431-436

Hajizadeh E, Mahootchi M, Esfahanipour A, et al (2019) A new nn-pso hybrid model for forecasting euro/dollar exchange rate volatility. Neural Computing and Applications 31(7):2063-2071

Hamid SA, Iqbal Z (2004) Using neural networks for forecasting volatility of s\&p 500 index futures prices. Journal of Business Research 57(10):1116-1125

Hoseinzade E, Haratizadeh S (2019) Cnnpred: Cnn-based stock market prediction using a diverse set of variables. Expert Systems with Applications 129:273-285

Karakoyun ES, Cibikdiken A (2018) Comparison of arima time series model and lstm deep learning algorithm for bitcoin price forecasting. In: The 13th multidisciplinary academic conference in Prague, pp 171-180

Kim HY, Won CH (2018) Forecasting the volatility of stock price index: A hybrid model integrating lstm with multiple garch-type models. Expert Systems with Applications 103:25-37

Kristjanpoller W, Minutolo MC (2015) Gold price volatility: A forecasting approach using the artificial neural network-garch model. Expert systems with applications 42(20):7245-7251

Krizhevsky A, Sutskever I, Hinton GE (2012) Imagenet classification with deep convolutional neural networks. Advances in neural information processing systems 25:1097-1105

Lawrence S, Giles CL, Tsoi AC, et al (1997) Face recognition: A convolutional neural-network approach. IEEE transactions on neural networks 8(1):98-113

Li H, Hong Y (2011) Financial volatility forecasting with range-based autoregressive volatility model. Finance Research Letters 8(2):69-76

Li W, Zhu L, Shi Y, et al (2020) User reviews: Sentiment analysis using lexicon integrated two-channel cnn-lstm family models. Applied Soft Computing 94:106,435

Livieris IE, Pintelas E, Pintelas P (2020) A cnn-lstm model for gold price timeseries forecasting. Neural computing and applications 32(23):17,351-17,360

Lu CJ, Lee TS, Chiu CC (2009) Financial time series forecasting using independent component analysis and support vector regression. Decision support systems 47(2):115-125

Maciel L, Gomide F, Ballini R (2016) Evolving fuzzy-garch approach for financial volatility modeling and forecasting. Computational Economics 

48(3):379-398

Mohammed GT, Aduda JA, Kube AO, et al (2020) Improving forecasts of the egarch model using artificial neural network and fuzzy inference system. Journal of Mathematics 2020:1-14

Naimy V, Haddad O, Fernández-Avilés G, et al (2021) The predictive capacity of garch-type models in measuring the volatility of crypto and world currencies. PloS one 16(1):e0245,904

Pérez-Rodríguez JV, Torra S, Andrada-Félix J (2005) Star and ann models: forecasting performance on the spanish "ibex-35" stock index. Journal of Empirical Finance 12(3):490-509

Quayyoum S, Khan MH, Shah SZA, et al (2020) Seasonality in crude oil returns. Soft Computing 24(18):13,547-13,556

Racine J (2001) On the nonlinear predictability of stock returns using financial and economic variables. Journal of Business \& Economic Statistics $19(3): 380-382$

Roh TH (2007) Forecasting the volatility of stock price index. Expert Systems with Applications 33(4):916-922

Sadik ZA, Date PM, Mitra G (2019) News augmented garch $(1,1)$ model for volatility prediction. IMA Journal of Management Mathematics 30(2):165185

Sagheer A, Kotb M (2019) Time series forecasting of petroleum production using deep lstm recurrent networks. Neurocomputing 323:203-213

Siami-Namini S, Tavakoli N, Namin AS (2018) A comparison of arima and lstm in forecasting time series. In: 2018 17th IEEE International Conference on Machine Learning and Applications (ICMLA), IEEE, pp 1394-1401

Sun H, Yu B (2020) Forecasting financial returns volatility: A garch-svr model. Computational Economics 55(2):451-471

Tang LB, Tang LX, Sheng HY (2009) Forecasting volatility based on wavelet support vector machine. Expert Systems with Applications 36(2):2901-2909

Tay FE, Cao L (2001) Application of support vector machines in financial time series forecasting. omega 29(4):309-317

Tomar A, Gupta N (2020) Prediction for the spread of covid-19 in india and effectiveness of preventive measures. Science of The Total Environment $728: 138,762$ 
Wang B, Wang J (2020) Forecasting hybrid neural network with variational learning rate and q-dscid synchronization evaluation for energy market. Soft Computing 24(22):16,811-16,828

Wang L, Feng C, Song Q, et al (2012) Efficient semiparametric garch modeling of financial volatility. Statistica sinica pp 249-270

Wang YH (2009) Nonlinear neural network forecasting model for stock index option price: Hybrid gjr-garch approach. Expert Systems with Applications $36(1): 564-570$

Wu CH, Lu CC, Ma YF, et al (2018) A new forecasting framework for bitcoin price with lstm. In: 2018 IEEE International Conference on Data Mining Workshops (ICDMW), IEEE, pp 168-175

Yang J, Nguyen MN, San PP, et al (2015) Deep convolutional neural networks on multichannel time series for human activity recognition. In: Twenty-fourth international joint conference on artificial intelligence

Yu L, Wang S, Lai KK (2009) A neural-network-based nonlinear metamodeling approach to financial time series forecasting. Applied Soft Computing $9(2): 563-574$

Zhang L, Wang J (2021) Forecasting global crude oil price fluctuation by novel hybrid e-sternn model and emccs assessment. Soft Computing 25(4):26472663

Zhao J, Mao X, Chen L (2019) Speech emotion recognition using deep 1d \& 2d cnn lstm networks. Biomedical Signal Processing and Control 47:312-323

Zhiqiang G, Huaiqing W, Quan L (2013) Financial time series forecasting using lpp and svm optimized by pso. Soft Computing 17(5):805-818

Zhong X, Enke D (2017) Forecasting daily stock market return using dimensionality reduction. Expert Systems with Applications 67:126-139 